\title{
Endoscopic totally extraperitoneal repair of bilateral inguinal hernias
}

\author{
M. T. T. Knook, W. F. Weidema*, L. P. S. Stassen†, R. U. Boelhouwer* and C. J. van Steensel* \\ Departments of Surgery, University Hospital Dijkzigt and *Ikazia Hospital, Rotterdam and †Reinier de Graaf Gasthuis, Delft, The Netherlands \\ Correspondence to: Dr M. T. T. Knook, Department of Surgery, University Hospital Dijkzigt, Dr Molewaterplein 40, 3015 GD Rotterdam, \\ The Netherlands
}

\begin{abstract}
Background: Recurrence rates associated with bilateral inguinal hernia repair with a giant prosthesis (Stoppa procedure) are low. Endoscopic totally extraperitoneal bilateral inguinal hernia repair with a giant prosthesis combines the low recurrence rate of the Stoppa repair and the advantages of minimally invasive surgery. The aim of this retrospective study was to investigate whether extraperitoneal bilateral inguinal hernia repair could be performed by the minimally invasive, totally extraperitoneal approach.

Methods: From February 1993 to January 1998, 98 patients with bilateral inguinal hernias underwent surgery. A polypropylene $30 \times 10 \mathrm{~cm}$ rectangular mesh or a $30 \times 10 / 15 \mathrm{~cm}$ 'slipmesh' was used. Followup, including a physical examination, of 96 per cent of patients was performed.

Results: Median operative time was $60 \mathrm{~min}$. Mostly minor intraoperative complications occurred. Conversion was required for two patients. Apart from one patient with a necrotic fasciitis who died from respiratory failure, only minor postoperative complications (10 per cent) occurred. Median hospital stay was 1 (range 1-21) days. Median recuperation time was 5 (range 1-22) days. Median follow-up (96 per cent) was 32 (range 7-57) months; there were six recurrences among 34 hernias in the group of 17 patients treated with $10 \times 30 \mathrm{~cm}$ mesh and two (1 per cent) in the group that received $30 \times 10 / 15 \mathrm{~cm}$ mesh (162 hernias in 81 patients).

Conclusion: The endoscopic approach for the Stoppa procedure for bilateral inguinal hernia repair is a reliable method with minor complications. It ensures a short recuperation time and the recurrence rate is low owing to adequate overlap of the hernial defect when a 'slipmesh' is used.
\end{abstract}

Paper accepted 26 May 1999

British Journal of Surgery 1999, 86, 1312-1316

\section{Introduction}

Some studies have reported an increased morbidity rate following bilateral inguinal hernia repair compared with unilateral hernia repair ${ }^{1,2}$; other authors disagree that the morbidity rate for bilateral inguinal hernia repair is higher when a tension-free repair is performed ${ }^{3-5}$. In view of the disadvantages of sequential unilateral hernia repair, such as higher total expenses and more sick leave, simultaneous repair is to be preferred ${ }^{1,4-7}$. From the standpoint of morbidity, an endoscopic technique for bilateral inguinal hernia repair is attractive since it is known to cause less postoperative pain and ensures more rapid recovery than conventional hernia repair ${ }^{1,2,8-10}$. Furthermore, the recurrence rate for conventional hernia repair without a mesh prosthesis remains high ${ }^{11}$. The prosthetic repair of bilateral inguinal hernias by the preperitoneal approach, as described by Stoppa and Warlaumont, has reduced recurrence rates significantly ${ }^{1,12,13}$.
Endoscopic totally extraperitoneal bilateral inguinal hernia repair with a giant prosthesis combines the low recurrence rate of the Stoppa prosthetic repair of groin hernias and the advantages of minimal invasive surgery $^{4,14-16}$.

\section{Patients and methods}

Ninety-eight patients with bilateral inguinal hernias at physical examination, who were fit for general anaesthesia and did not have an infection of the abdominal wall, underwent surgery. Peroperative hernia classification was performed as proposed by Nyhus ${ }^{12}$. All operations were performed by three staff surgeons experienced in endoscopic surgery. The procedure used was standardized for all cases. The essential steps of total extraperitoneal repair have been described by Liem et al. ${ }^{17}$.

For the first 17 cases of this study a $10 \times 30 \mathrm{~cm}$ polypropylene prosthesis (Prolene; Ethicon, Edinburgh, 
UK) was used. However, a relatively large number of medial recurrences apparently occurred owing to insufficient overlap of the medial orifice when using the $10 \times 30 \mathrm{~cm}$ prosthesis; therefore a $10 / 15 \times 30 \mathrm{~cm}$ 'slipmesh' polypropylene prosthesis was used for the subsequent 81 patients. The 'slipmesh' (Fig. 1) is a tailored mesh of $15 \times 30 \mathrm{~cm}$. The 'slip' can be pulled into a position which results in better coverage of the area of the direct hernias.

When intraoperative complications led to the need for conversion to another endoscopic or an open technique, the procedure was chosen by the operating surgeon.

After operation patients were mobilized directly and were allowed to leave the hospital as soon as they felt able as long as postoperative complications requiring clinical care had not occurred.

The first 54 patients were seen at regular 3-month intervals during the first year after operation and then annually by the authors. The other patients (43) were seen 1 week after operation and, if no problems occurred, further follow-up was done on demand. For the present study initial data and data recorded during follow-up were collected from patient records. Operative time was defined as the time from the first incision to the last suture. Intraoperative complications (diffuse haemorrhage or bleeding from an epigastric vessel, peritoneal defects), postoperative complications (large haematomas of the abdominal wall, seroma, paraesthesia, wound infection, urine retention, mesh infection, pneumopericardium) and conversion of the totally extraperitoneal operation to an endoscopic transabdominal or open procedure were noted. Length of hospital stay (number of days in hospital after operation), recuperation time (number of days needed for recovery before returning to work or full daily activities) and recurrence of a hernia were assessed. To complete this study with up-to-date recurrence rates, all patients were approached by mail or telephone and asked to report to the

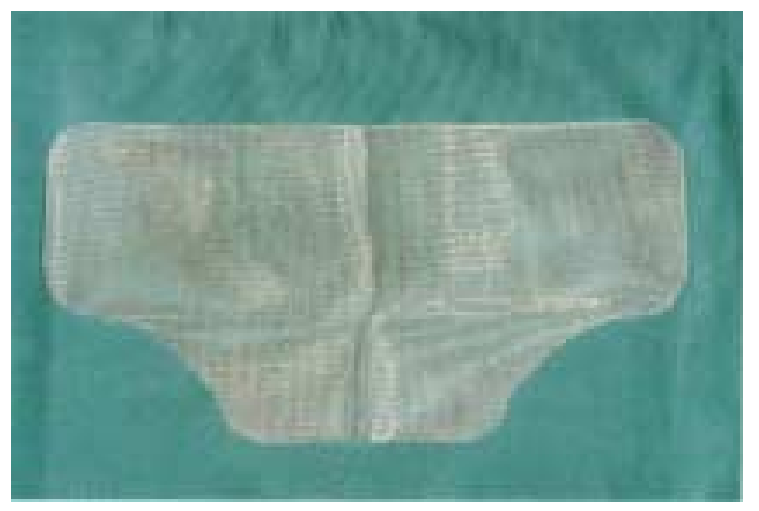

Fig. 1 'Slipmesh' prosthesis $(10 / 15 \times 30 \mathrm{~cm})$ outpatient department for a physical examination. Appointments were made at two fixed dates at which patients (96 per cent) had a thorough physical examination performed by a staff surgeon and a senior resident (both authors of this manuscript). At this final check-up, initial data missing from patient records were collected and recurrence of the hernia was evaluated by means of a thorough physical examination.

\section{Results}

From February 1993 to January 1998, 98 patients (96 men and two women) were operated on. Their age at surgery ranged from 27 to 86 (median 60) years. Hernia localization, classified according to Nyhus, is given in Table 1. In 75 patients a primary bilateral inguinal hernia was diagnosed. In 14 patients the hernia was primary on one side and recurrent on the other, and in another nine patients the hernia was recurrent on both sides. The recurrent hernias had all developed after previous conventional hernia repair without the use of prosthetic material. For dissection a peritoneal dissection balloon was used in 80 cases, leading to peritoneal tears during dissection in 14 (18 per cent). In the other 18 patients dissection was performed with the endoscope leading to peritoneal tears in all instances. The median operative time was 60 (range 20-120) min.

Intraoperative and postoperative complications are summarized in Table 2. In one patient the epigastric vessels were injured such that clips were needed to stop the bleeding. In 30 of 32 patients with minor peritoneal tears the peritoneal defect was closed with a Polydioxanone-S (Ethicon) running suture or endoloop. Twice this was not possible: in one patient dissection difficulties owing to leakage of gas to the abdominal cavity made it necessary to convert the procedure to a transabdominal preperitoneal (TAPP) repair with a $10 \times 15 \mathrm{~cm}$ prosthesis on each side; in the other patient the procedure was continued using the open Stoppa approach.

Postoperative complications occurred in 10 per cent of the patients, most of which were minor. Small haematomas which resolved spontaneously accounted for another 11 per

Table 1 Classification according to Nyhus of 98 patients with a bilateral inguinal hernia

\begin{tabular}{lrlrll}
\multicolumn{7}{c}{ Nyhus classification } \\
II, II & 20 & IIIA, IIIA & 19 & IIIB, IIIB & 9 \\
II, IIIA & 11 & IIIA, IIIB & 10 & IIIB, IV & 3 \\
II, IIIB & 6 & IIIA, IV & 8 & IV, IV & 9 \\
II, IV & 3 & & & &
\end{tabular}

NII, Indirect hernia; NIIIA, direct hernia; NIIIB, combined hernia; NIV, recurrent hernia 
Table 2 Complications among 98 patients who underwent endoscopic totally extraperitoneal repair of a bilateral inguinal hernia

\begin{tabular}{lc} 
& No. of patients \\
\hline Peroperative complications & \\
Peritoneal tears & $32(33)$ \\
Conversion & $2(2)$ \\
TAPP repair & $1(1)$ \\
Open Stoppa & $1(1)$ \\
Epigastric vessel laceration & $1(1)$ \\
Postoperative complications & \\
Haematoma & $11(11)$ \\
Mesh infection & $2(2)$ \\
Urine retention* & $2(2)$ \\
Pneumopericardium & $1(1)$ \\
Seroma & $1(1)$ \\
Cystitis & $2(2)$ \\
Temperature (of unknown origin) & $1(1)$ \\
Paraesthesia & $2(2)$
\end{tabular}

Values in parentheses are percentage. *This occurred in a patient with a pre-existing urethral stricture. TAPP, transabdominal preperitoneal

cent of 'postoperative complications'. Mild paraesthesia of the medial thigh in two patients was probably due to injury of the ilioinguinal nerve; at 3 months it had completely disappeared in both patients. The seroma needed fineneedle aspiration and the cystitis was treated with antibiotics. One mesh infection was treated successfully by ultrasonographically guided drainage and the administration of intravenous antibiotics; at follow-up no signs of ongoing infection were present. The other patient with a mesh infection suffered a dramatic necrotizing fasciitis which, although cured by extensive abdominal resection, led to his death from acute respiratory distress syndrome (ARDS) and pulmonary insufficiency.

Median hospital stay was 1 (mean 2, range 1-21) day. The patient with the infected mesh that was treated successfully and a patient who underwent dilatation of a urethral stricture were hospitalized for 14 and 21 days respectively. The patient who died from ARDS due to necrotizing fasciitis was in hospital for 21 days. The 30-day hospital mortality rate was thus 1 per cent. One patient with a raised temperature of unknown origin was hospitalized for analysis for 2 weeks until the temperature resolved spontaneously. Patients returned to work or full daily activities after a median period of 5 (mean 7, range 1-22) days.

Ninety-four of 98 patients responded to the postal questionnaire and, in some cases, subsequent telephone request; therefore follow-up with a physical examination was accomplished in 96 per cent. Median follow-up was 32 (mean 30, range 7-57) months.

Eight recurrences were diagnosed in seven patients. In six patients a unilateral recurrent inguinal hernia was seen at physical examination. In one patient a bilateral recurrent hernia was diagnosed. Five patients had noticed bulging; the other two patients were asymptomatic. Six of these recurrences occurred within the first year of surgery for a primary inguinal hernia. Six of the patients with a recurrence belonged to the group of 17 patients ( 34 hernias; recurrence rate 18 per cent) who received the $10 \times 30 \mathrm{~cm}$ rectangular prosthesis. Of the 81 patients ( 162 hernias) with a $10 / 15 \times 30 \mathrm{~cm}$ slip prosthesis, two (1 per cent) suffered a recurrence. Reintervention was performed in five cases (six hernias) by means of a TAPP procedure. This reintervention allowed the cause of the recurrence to be investigated. In all cases the recurrent hernia was located medially, and thus was probably due to insufficient overlap of the defect by the rectangular mesh or inadequate positioning of the 'slipmesh'. The two fully asymptomatic patients preferred to postpone the operation until complaints developed.

\section{Discussion}

Both the use of a prosthetic mesh to create a tension-free repair and the endoscopic technique have gained popularity in inguinal hernia surgery ${ }^{3,4,9}$. Stoppa and others have used the preperitoneal subumbilical approach to the retrofascial space since 1969. Advantages of this approach were the ease of separation of the retrofascial cellular space, direct access to the posterior inguinal structures, clear understanding of hernial lesions, and clear exposure of the musculopectineal opening. Furthermore, in their opinion, the retrofascial space is a natural site for prostheses to reinforce the transversalis fascia.

Although several promising reports on endoscopic inguinal hernia repair have been published ${ }^{2,8,18,19}$, reports on endoscopic bilateral inguinal hernia repair are scarce ${ }^{4,10}$. As a rule these bilateral procedures are small in number and are described as part of a study on endoscopic unilateral inguinal hernia repair ${ }^{8,12,18,20}$. The technique most often used for these bilateral hernias is the TAPP technique with two meshes of $10 \times 15 \mathrm{~cm}$ or one single large mesh ${ }^{7}$. The totally extraperitoneal procedure, however, is the preferred technique in most situations, as it is less difficult to position a large mesh ${ }^{18,21,22}$.

The choice of a single large prosthesis might reduce the problem of mesh migration and the risk of insufficient overlap of the medial defect ${ }^{10,14,21}$. It is important to cover the areas of both indirect and direct (potential) hernias of the inguinal floor in all cases to reduce hernia recurrence ${ }^{21}$. In this study of bilateral inguinal hernia repair the Stoppa procedure was adopted in combination with an endoscopic approach. Stoppa and Warlaumont ${ }^{13}$ reported a recurrence rate of 1.4 per cent using their technique. In their opinion the recurrences that did occur resulted from insufficient 
mesh size or shape since the recurrences passed through insufficient fascia under the lower edge. The same problem was encountered using the rectangular mesh in the present study and therefore a switch was made to the 'slipmesh' prosthesis which resulted in a lower recurrence rate (1 per cent).

In the present study of totally extraperitoneal repair of bilateral inguinal hernias the occurrence of intraoperative and postoperative complications was comparable to that of other reports on endoscopic inguinal hernia repair $8,9,18,21,22$. Nevertheless, two serious complications did occur. Although one of these patients did not suffer any late problems from mesh infection, the second patient died from respiratory failure during treatment of necrotizing fasciitis in the intensive care unit. For this reason selective indications for repair with prostheses (no abdominal wall infections, no other active infections), irrespective of the procedure, and careful attention to the prevention of septic complications of both conventional and endoscopic repairs are essential ${ }^{13}$. Antibiotics were not administered in these patients; these mesh infections were considered to be a coincidence in this subgroup of patients as mesh infections have not occurred at all in the (more than 1000) other hernia repairs performed by the authors. Operative time was comparable to, or even shorter than, that for conventional surgery.

The short hospital stay and rapid recuperation in this group are comparable to those of earlier reports ${ }^{2,4,14}$. Simultaneous repair of bilateral hernias which, according to some authors, is controversial in the event of open inguinal hernia repair, does not lead to extra hospitalization or longer recuperation when the endoscopic approach is applied ${ }^{4,14,16}$. The wide range in the length of hospital stay and the recuperation time may be explained by the extended clinical treatment of a mesh infection in one patient, the surgical treatment of an urethral stricture in another and the conversion to an open repair in two patients.

Many recurrences are not noticed by the patient and will therefore not be reported in questionnaires or during telephone interviews ${ }^{4,9,11,16}$. The recurrence rate was high (18 per cent) for the 17 patients ( 34 hernias) in whom a rectangular mesh was used. In four of these six patients reintervention took place. All of these recurrences were found to be medial. Probably the rectangular shape of this prosthesis does not give adequate coverage of the medial defects. With use of the 'slipmesh', which ensures more complete overlap of medial defects, the recurrence rate dropped to 1 per cent ${ }^{14}$. Recurrence in this case is most probably still due to inadequate dissection or inadequate positioning of the mesh: the prosthesis was not found in the right position medially at reintervention for the recurrent hernia. Adequate dissection and mesh positioning when using an appropriate mesh size should further decrease recurrence rates.

The results of this procedure as shown are promising. It should, however, be emphasized that a relatively long learning curve ${ }^{17}$ is to be expected since both the introduction and the positioning of the mesh prosthesis are quite difficult.

Although this procedure is feasible the question remains as to whether it is also reasonable. Increased costs of the procedure are often mentioned as a negative feature. The decreased costs due to shorter sick leave and fewer readmissions for hernia recurrences may compensate for the higher costs of the endoscopic procedure.

\section{References}

1 Baeten CG, van Geldere D, Kootstra G. Current treatment of bilateral inguinal hernias using preperitoneal Marlex mesh prosthesis. Ned Tijdschr Geneeskd 1990; 134: 535-7.

2 McKernan JB, Laws HL. Laparoscopic repair of inguinal hernias using a totally extraperitoneal prosthetic approach. Surg Endosc 1993; 7: 26-8.

3 Amid PK, Shulman AG, Lichtenstein IL. Simultaneous repair of bilateral inguinal hernias under local anesthesia. Ann Surg 1996; 223: 249-52.

4 Geis WP, Malago M. Laparoscopic bilateral inguinal herniorrhaphies: use of a single giant preperitoneal mesh patch. Am Surg 1994; 60: 558-63.

5 Stott MA, Sutton R, Royle GT. Bilateral inguinal hernias: simultaneous or sequential repair? Postgrad Med f 1988; 64: $375-8$.

6 Miller AR, van Heerden JA, Naessens JM, O'Brien PC. Simultaneous bilateral hernia repair. A case against conventional wisdom. Ann Surg 1991; 213: 272-6.

7 Serpell JW, Johnson CD, Jarrett PE. A prospective study of bilateral inguinal hernia repair. Ann R Coll Surg Engl 1990; 72: 299-303.

8 Felix EL, Michas CA, Gonzalez MH Jr. Laparoscopic hernioplasty: why does it work? Surg Endosc 1997; 11: 36-41.

9 Liem MSL, van der GraafY, van Steensel CJ, Boelhouwer RU, Clevers GJ, Meijer WS et al. Comparison of conventional anterior surgery and laparoscopic surgery for inguinal hernia repair. N Engl F Med 1997; 336: 1541-7.

10 Champault GG, Rizk N, Catheline JM, Turner R, Boutelier P. Inguinal hernia repair: totally preperitoneal laparoscopic approach versus Stoppa operation: randomized trial of 100 cases. Surg Laparosc Endosc 1997; 7: 445-50.

11 Hay JM, Boudet MJ, Fingerhut A, Poucher J, Hennet H, Habib E et al. Shouldice inguinal hernia repair in the male adult: the gold standard? A multicenter controlled trial in 1578 patients. Ann Surg 1995; 222: 719-27.

12 Nyhus LM. Individualization of hernia repair: a new era. Surgery 1993; 114: 1-2.

13 Stoppa RE, Warlaumont CR. The preperitoneal approach and 
prosthetic repair of groin hernias. In: Nyhus LM, Condon RE, eds. Hernia. Philadelphia, Pennsylvania: JB Lippincott, 1995: 188-210.

14 Deans GT, Wilson MS, Royston CM, Brough WA. Laparoscopic 'bikini mesh' repair of bilateral inguinal hernia. Br 7 Surg 1995; 82: 1383-5.

15 Liem MS, van der Graaf Y, Zwart RC, Geurts I, van Vroonhoven TJ. A randomized comparison of physical performance following laparoscopic and open inguinal hernia repair. The Coala Trial Group. Br 7 Surg 1997; 84: 64-7.

16 Velasco JM, Gelman C, Vallina VL. Preperitoneal bilateral inguinal herniorrhaphy: evolution of a technique from conventional to laparoscopic. Surg Endosc 1996; 10: 122-7.

17 Liem MSL, van Steensel CJ, Boelhouwer RU, Weidema WF, Clevers GJ, Meijer WS et al. The learning curve for totally extraperitoneal laparoscopic inguinal hernia repair. Am 7 Surg 1996; 171: 281-5.

18 Felix EL, Michas CA, Gonzales MH Jr. Laparoscopic hernioplasty: TAPP vs TEP. Surg Endosc 1995; 9: 984-9.

19 Panton ONM, Panton RJ. Laparoscopic hernia repair. Am 7 Surg 1994; 167: 535-7.

20 Topal B, Hourlay P. Totally preperitoneal endoscopic inguinal hernia repair. Br 7 Surg 1997; 84: 61-3.

21 Phillips EH, Arregui M, Carroll BJ, Corbitt J, Crafton WB, Fallas MJ et al. Incidence of complications following laparoscopic hernioplasty. Surg Endosc 1995; 9: 16-21.

22 Ramshaw BJ, Tucker JG, Conner T, Mason EM, Duncan TD, Lucas GW et al. A comparison of the approaches to laparoscopic herniorrhaphy. Surg Endosc 1996; 10: 29-32. 